\title{
GRB POTENTIAL OF ESA GAIA
}

\author{
R. Hudec ${ }^{1,2}$ and V. Šimon ${ }^{1}$
}

\begin{abstract}
The potential of GRB analyses (including highly redshifted objects) with ESA mission Gaia is briefly addressed.
\end{abstract}

\section{Introduction}

ESA Gaia satellite, with expected launch date in 2013, is an ambitious mission to chart a three-dimensional map of our Galaxy, the Milky Way, in the process revealing the composition, formation and evolution of the Galaxy(Perryman 2005, 2006). Gaia will provide unprecedented positional and radial velocity measurements with the accuracies needed to produce a stereoscopic and kinematic census of about one billion stars in our Galaxy and throughout the Local Group. This amounts to about 1 per cent of the Galactic stellar population. Combined with astrophysical information for each star, provided by on-board multi-colour photometry, these data will have the precision necessary to quantify the early formation, and subsequent dynamical, chemical and star formation evolution of the Milky Way Galaxy. Additional scientific products include detection and orbital classification of tens of thousands of extra-solar planetary systems, a comprehensive survey of objects ranging from huge numbers of minor bodies in our Solar System, through galaxies in the nearby Universe, to some 500000 distant quasars. It will also provide a number of stringent new tests of general relativity and cosmology (http://gaia.esa.int).

The payload consists of a single integrated instrument the design of which is characterised by: A dual telescope concept, with a common structure and a common focal plane. Both telescopes are based on a three-mirror anastigmat (TMA) design. Beam combination is achieved in image space with a small beam combiner. Silicon-carbide (SiC) ultra-stable material is used for mirrors and telescope

\footnotetext{
${ }^{1}$ Astronomical Institute, Academy of Sciences of the Czech Republic, 25165 Ondřejov, Czech Republic

2 Czech Technical University in Prague, Faculty of Electrical Engineering, Prague, Czech Republic
} 
structure A highly robust measurement system for the Basic Angle between the two telescopes' pointing directions A large common focal plane with an array of 106 CCDs. The large focal plane also includes areas dedicated to the spacecraft's metrology and alingment measurements. The satellite will be placed on the Lissajous-type orbit around L2 (http://gaia.esa.int).

To study the optical counterparts of gamma-ray bursts (GRBs), Gaia will have several advantages. First, it will be a deep limiting magnitude of 20 mag (Jordi \& Carrasco 2007), much deeper than most of the previous studies and global surveys. Secondly, the time period covered by Gaia observations, i.e. 5 years, will also allow some studies requiring long-term monitoring. The most important advantage of Gaia for GRB studies will be the color (spectral) resolution. This will allow some detailed studies involving analysis of the color and spectral changes not possible before. The details of studies of the optical counterparts of high-energy sources are described in detail in the dedicated sub-workpackages within the workpackage Specific objects studies within the Gaia CU7 (Hudec \& Šimon 2007a,b).

\section{Photometry}

The Optical Transients (OTs) and Optical Afterglows (OAs) of GRBs usually reach their peak optical luminosity in the initial phase, shortly (several minutes) after the gamma-ray emission which typically lasts from a fraction of second to several minutes. In the later, much longer phase which can last for several (even more than 10) days, the OAs usually display a characteristic power-law fading profile (if a logarithm of the time interval between the start of the GRB and the given observation is used, and the brightness is measured in magnitudes). A sequence of observations mapping this OA light curve is therefore necessary. According to Zhang (2007), most of OAs are fainter than about 18 mag already about 1 day after the GRB, although some of them can be even brighter than 14 mag in the early phase. Gaia is therefore definitely able to detect these OAs in their early phase. However, the sampling provided by Gaia is not optimal, hence only rarely we can expect detection of OA of GRB based only on this type of data.

\section{Spectro-photometry/low-dispersion spectroscopy}

The Gaia instrument consists of two low-resolution fused-silica prisms dispersing all the light entering the field of view (FOV). Two CCD strips are dedicated to photometry, one for blue photometer (BP) and one for red photometer (RP). Both strips cover the full astrometric FOV in the across-scan direction. All BP and RP CCDs are operated in TDI (time-delayed integration) mode. CCDs have 4500 (for BP) or 2900 (for RP) TDI lines and 1966 pixel columns $(10 \times 30$ micron pixels). The spectral resolution is a function of wavelength as a result of the natural dispersion curve of fused silica. The BP and RP dispersers have been designed in such a way that $\mathrm{BP}$ and $\mathrm{RP}$ spectra have similar sizes (on the order 


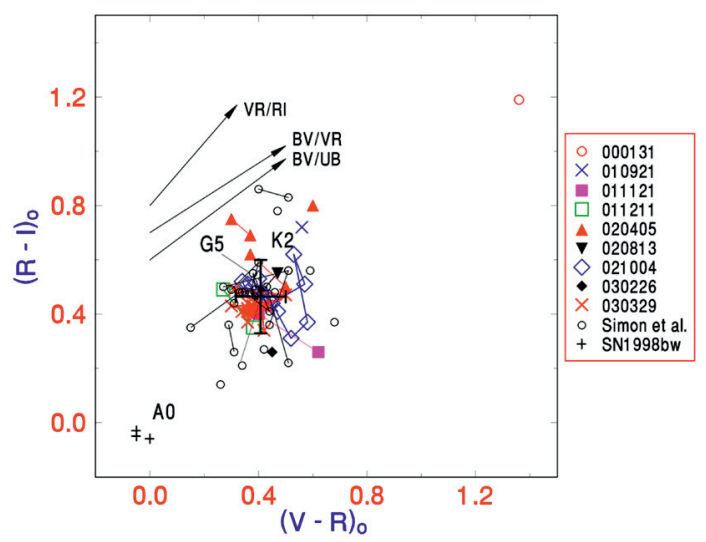

Fig. 1. Example of the color-color diagram of OAs of long GRBs. The data for the time interval $<10.2 \mathrm{~d}$ after the burst in the observer frame and corrected for the Galactic reddening are displayed. Multiple indices of the same OA are connected by the lines for convenience. The mean colors (centroid) of the whole ensemble of OAs (except for GRB 000131 and SN 1998bw) are marked by the large cross. The representative reddening paths for $E_{B-V}=0.5 \mathrm{mag}$ and positions of the main-sequence stars are also shown. Adapted from Šimon et al. (2001, 2004a).

of 30 pixels along scan). BP and RP spectra will be binned on-chip in the acrossscan direction; no along-scan binning is foreseen. $\mathrm{RP}$ and $\mathrm{BP}$ will be able to reach the object densities in the sky of at least 750000 objects $\operatorname{deg}^{-2}$. Albeit the dispersion is low, the major strength of Gaia for many astrophysical fields will be the spectrophotometry, as the low dispersion spectra may be transferred to numerous well-defined color filters. We have shown (Šimon et al. 2001, 2004) that the individual OAs of GRBs display quite specific and remarkably similar color indices with negligible changes during the first several days after the GRB (an example of such a color-color diagram is shown in Fig. 2). This feature is important for distinguishing OAs from other types of astrophysical objects. This suggests that although OAs possess a large range of redshifts $z$, they display very similar spectra in the observer frame for $z<3.5$. This gives us a hope to resolve whether an optical event is related to a GRB even without available gamma-ray detection.

\section{Strategy and detection rate}

The Gaia instruments will map the entire sky with deep limits. The duration of most of OA is about 10-20 days in the observer frame, hence they are likely to be detected by Gaia during its scans even without rapid pointing at the GRB position. However, this assumes that they will occur in the FOV of Gaia telescopes. As already indicated, the OA can be recognized according to several features even 
without information on the time profile. The following features appear to be important: (1) unique color indices, (2) rapid rise (a new object appears between two scans), (3) host galaxy of the GRB at the position of OA - this galaxy can be detected by ground-based observations later. We note that even search for orphan afterglows will be possible with Gaia. The missing gamma-ray emission, with only an OA remaining, can also suggest this important event. Gamma-ray emission from many GRBs remains unobservable because the jet is not pointing to the observer, but the late-time OA is less beamed and can reach us (Rhoads 1997; Rossi et al. 2008). Also failed GRBs are possible contributors to the population of orphan afterglows (Huang et al. 2002).

The estimated Gaia detection rate for OAs of GRBs, including orphans, is expected to be up to $\sim 100$ in the whole Gaia lifetime ( 5 years). This low rate is due to small FOV of the Gaia telescopes ( $\sim 0.36 \mathrm{deg}^{2}$ each). Higher detection rate is expected in plate Low-Dispersion Spectroscopy (LDS) surveys (due to much larger FOV) in which analogous strategies (e.g. high-redshift triggers) can be applied.

\section{Highly redshifted Universe}

Some GRBs may be at large distances and hence highly redshifted. The Gaia mission will be able to study highly redshifted triggers. The redshifted Lyman alpha line/break can be used to measure the value of $z$. This was e.g. the idea of the proposed Joint Astrophysics Nascent Universe Satellite (JANUS) Small Explorer (SMEX) Space Mission (Fox 2010) with coverage range of 0.7-1.7 microns (Gaia RP has a coverage of 0.65-1.0 microns). GRBs are located at cosmological distances, often with $z>0.5$ (e.g. Robertson \& Ellis 2012). The Lyman break is therefore shifted to the optical band for the objects at $z$ larger than about 3.5. This break manifests itself as a sharp decrease of the flux in the blue part of the spectrum. Such a feature is prominent in the smooth spectral profile of OA. This OA will therefore appear shifted from its true position because of the lack of its blue part of the spectrum. A comparison of the accurate position of the OA obtained by Gaia in the astrometric mode with the blue edge of its spectrum can be used for an easy resolving the objects occurring in our Galaxy from those located at cosmological distances. Also the determination of $z$, hence of its distance necessary for the determination of its luminosity, will be possible.

\section{Conclusions}

We conclude that the ESA Gaia satellite will contribute to scientific investigations of GRBs. In the field of GRB study, Gaia advantages can be briefly summarized as follows. (1) Unique chance to provide early or simultaneous LDS for GRBs (so far LDS mostly late) (2) Chance to recognize/classify OAs and OTs of GRBs using LDS and/or color information even in their later phases and without known 
GRB (3) Chance to detect/study orphan OAs of GRBs and (4) Chance of redshift estimation up to $\sim 7$.

The Czech participation in the ESA Gaia project was supported by the PECS project 98058 . The scientific part of the study is related to the grant 102/09/0997 provided by the Grant Agency of Czech Republic (GA CR). The analyzes of spectral plates are supported by GA CR grant Digitizing Astronomical Plate Archives and Investigation of Celestial Sources in Digitized Plate Archives 13-39464J.

\section{References}

Fox, D., 2010, BAAS, 42, 573

Huang, Y.F., Dai, Z.G., \& Lu, T., 2002, MNRAS, 332, 735

Hudec, L., 2007, Algorithms for spectral classification of stars, BSc. Thesis (Charles University, Prague)

Hudec, R., \& Šimon, V., 2007a, Specific object studies for cataclysmic variables and related objects ESA Gaia Reference Code GAIA-C7-TN-AIO-RH-001-1

Hudec, R., \& Šimon, V., 2007b, Specific object studies for optical counterparts of high energy sources. ESA Gaia Reference Code GAIA-C7-TN-AIO-RH-002-1

Jordi, C., \& Carrasco, J.M., 2007, ASP Conf. Ser., 364, 215

Perryman, M., 2005, ASP Conf. Ser., 338, 3

Perryman, M., 2006, Gaia overall science goals, http://sci.esa.int/gaia/

Rhoads, J., 1997, ApJ, 487, L1

Robertson, B.E., \& Ellis, R.S., 2012, ApJ, 744, 95

Rossi, E.M., Perna, R., \& Daigne, F., 2008, MNRAS, 390, 675

Simon, V., Hudec, R., Pizzichini, G., \& Masetti, N., 2001, A\&A, 377, 450

Šimon, V., Hudec, R., Pizzichini, G., \& Masetti, N., 2004, AIP Conf. Proc. 727, 487

Zhang, B., 2007, ChJAA, 7, 1 
